\title{
Design of Pulse Oximeter Solution for Conscious Rodents
}

\author{
Lauren Elizabeth Lester, Elliott Dirr, \& Kevin Otto \\ University of Florida
}

Faculty Mentor: Kevin Otto, J. Crayton Pruitt Department of Biomedical Engineering

\begin{abstract}
Neuromodulation can be used to control organ function through exogenous augmentation of neural activity via targeted delivery of an electrical stimulus. Recently, neuromodulation has been a topic of investigation to treat many illnesses and conditions including Parkinson's disease, epilepsy, obesity, chronic pain, type 1 diabetes, and hypertension. During neuromodulatory treatments, it is important to measure both the physiological response of the target system as well as any off-target systems that may be engaged from the delivered stimulus. Stimulation of the vagus nerve, a common target of neuromodulatory therapies, causes a vasovagal response which results in decreased cardiac rate and arteriolar dilatation. It is possible to measure these physiological parameters in anesthetized rats, but it is a challenge in conscious, moving rats because of motion artifact introduced to the sensors. By creating a noninvasive, stabilized pulse oximeter that measures the blood oxygenation waveform, one can analyze the heart rate in awake behaving animals. This study reports the design of a heart rate monitor used to monitor effects of heart rate variability during neuromodulation experiments. Design of this device included testing different sensors, methods of attaching to the rodent, microcontrollers, and wireless communication modules.
\end{abstract}

Keywords: Pulse Oximetry, Neuromodulation, Noninvasive

\section{Introduction}

Since the early 1900s, the relationship between heart rate and the vagus nerve has been studied. Early findings indicated that increased signaling through the vagus nerve results in the lowering of the heart rate and increase in heart rate variability (Hering, 1910). Conversely, changes in heart rate and respiration rate can be indicative of changes of parasympathetic tone (van Ravenswaaij-Arts, 1993). Changes in heart rate variability can also tell us about the risk for sudden cardiac death in patients and mirrors the health of the cardiovascular system (van Ravenswaaij-Arts, 1993). Furthermore, changes in heart rate and blood pressure cause a change in organ perfusion, confounding experiments on organ modulation. Exogenous changes to parasympathetic tone, such as through electrical stimulation of the cervical vagus nerve [i.e. vagus nerve stimulation (VNS)], can cause changes in these physiological parameters (Buschman, 2006). 
The vagus nerve, described as the "wanderer nerve", is the longest cranial nerve and connects to all the visceral organs. Currently, VNS is approved by the FDA to treat a number of diseases such as depression and epilepsy. Side effects of this treatment can include headaches, shortness of breath, insomnia (Schachter, 2002), and decreased heart rate (Buschman, 2006). Recently, targeted vagus nerve stimulation has been investigated to modulate organs of interest to treat diseases such as type 1 diabetes (Dirr, 2019). In this approach, an electrode is placed on a distal branch of the vagus nerve allowing direct modulation of the pancreas while minimizing signaling to other organs and therefore eliminating side effects. When using a targeted method of vagus nerve stimulation such as subdiaphragmatic stimulation, heart rate can be measured to assess effects on change in systemic parasympathetic tone. It is possible to measure these physiological parameters in anesthetized rats (Payne et al, 2019), but there is a need for a cost effective, noninvasive pulse oximeters that filter motion artifacts well enough to be used on awake, conscious rodents.

Here the report of a pulse oximeter system that outputs an oxygen saturation waveform in which the heart rate can be identified in an awake rodent is given. This instrument is designed to be non-invasive and minimally cumbersome for the animal to wear. This device is pertinent to vagal nerve stimulation studies to determine if stimulus is affecting the targeted systems of the body according to the electrode location.

\section{Methods}

\section{Analysis and Assembly of Pulse Oximeter}

A pulse oximeter system was constructed to allow for wireless recordings in awake animals. NIH and Institutional guidelines for the care and use of animals (protocol numbers 201709797, 201708217) were followed in the performance of the experiments. Male Lewis rats over $400 \mathrm{~g}$ were used in experiments to evaluate device design criteria. Since the goal of this project was to produce a solution that can be used in multiple neuromodulation studies, two distinct rat models that were implanted with electrodes on different subdiaphragmatic branches of the vagus nerve were used in this study.

This evaluation used Male Lewis Rats that had a chemically induced Type 1 Diabetes (T1D) model through streptozotocin (STZ) injection. Method of attachment to the rodent tested two different aspects: location of where the sensor would be placed, and how long the device could stay on the rodent. Three locations were tested for the most stable and best heart rate waveform, 
including the neck, tail and foot. To determine the best location for sensor placement, unshaven awake diabetic rats were equipped with the MAX30100 (Maxim Integrated, San Jose, CA) pulse oximetry module held to their body in various location. Data were recorded and analyzed for heart rate containing signals. A Fast Fourier Transform (FFT) was computed to identify power within the expected heart rate band. The average heart rate for rats range between $200-400$ beats per minute (BPM), according to literature (Lawler, 1985), which is significantly faster than humans'. The expected heart rate band would then be between $3.33 \mathrm{~Hz}$ and $6.66 \mathrm{~Hz}$.

After selecting a body location with the highest power in the BPM band, a custom circular clip was made to hold the sensor onto rodents with an average weight of 400-600 g (Figure 1). The clip was made from Polylactic Acid (PLA), printed from a Ultimaker 2+ (Ultimaker, Waltham, MA) 3D printer, and designed using SolidWorks. A PulseSensor (World Famous Electronics LLC., Brooklyn, NY) pulse oximetry module was adhered to the bottom of the chassis to contact the rodent's neck to perform reflective based photoplethysmography. This

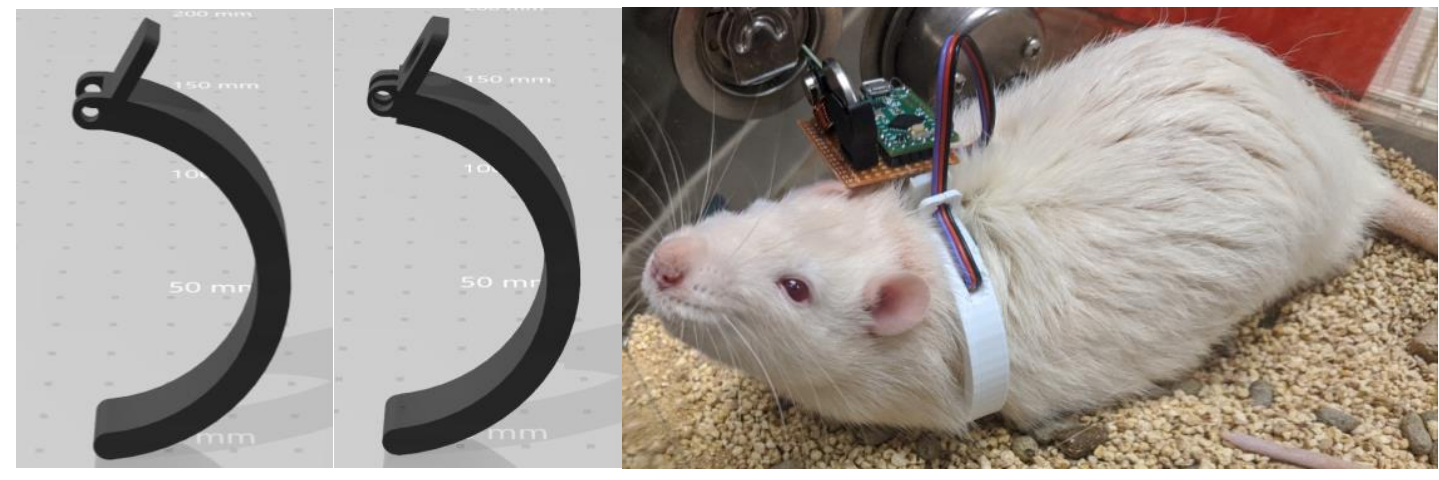

Figure 1. Left. A rendering of each part of the chosen clip design. Right. The final device with attached sensor, microcontroller, battery, and transmitter being worn by an awake rodent. 
signal was fed to a 10-bit ADC on a Teensy LC (PRJC, Sherwood, OR) and powered from a 3V cell battery. A FS1000a transmitter and receiver (FSI, Lombard, IL) was used to send data wirelessly at $433 \mathrm{MHz}$ through radio frequency transmission (Table 1).

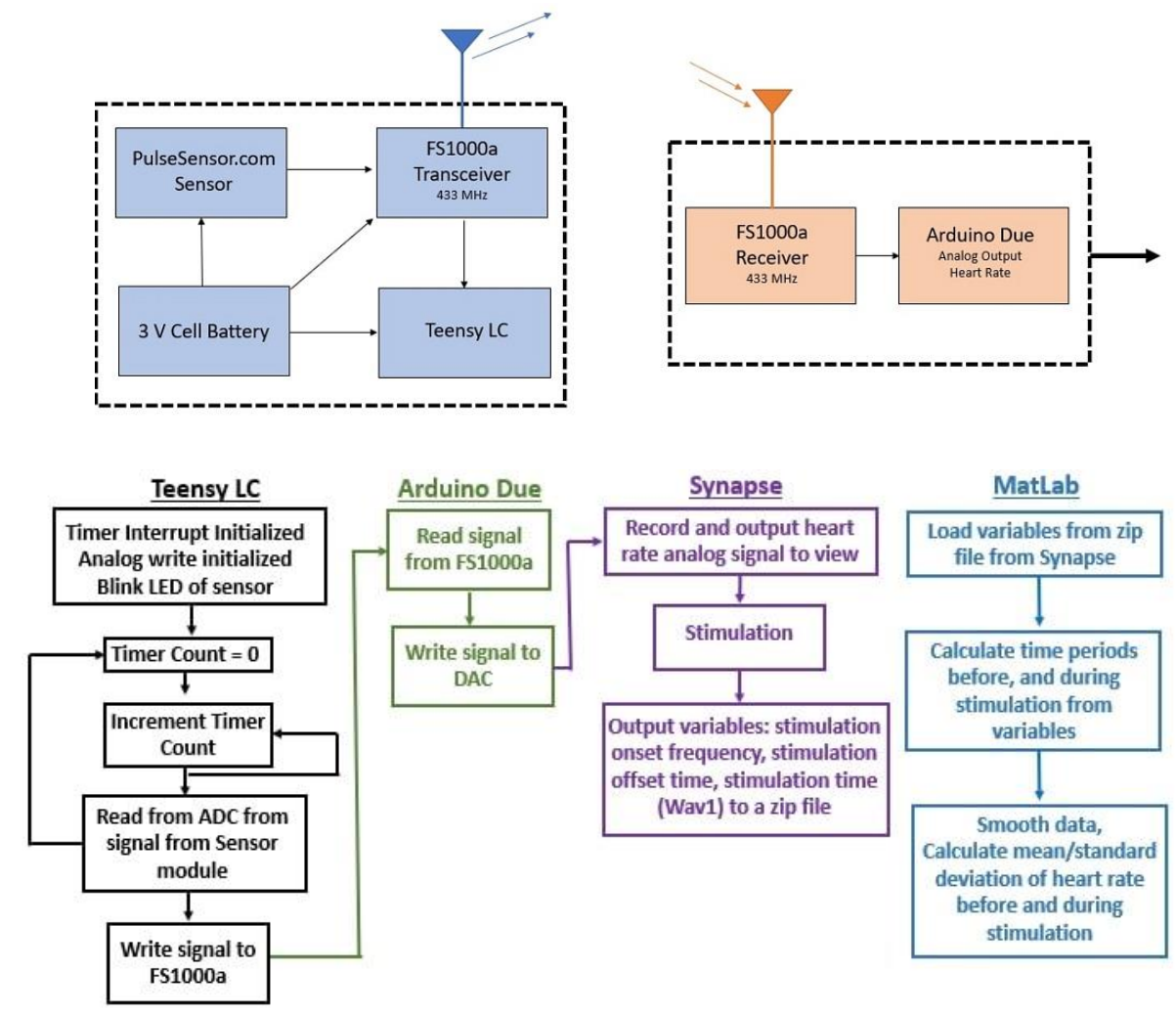

Figure 2. Block Diagram (Top) and software flow chart (Bottom) for the final design. The Teensy LC controlled the PulseSensor and transceiver and was powered by the $3 \mathrm{~V}$ cell battery. The Arduino Due controlled the receiver and output an analog signal to the RZ5D real time processor.

Custom Arduino software was written to measure pulse oximetry at $500 \mathrm{~Hz}$ and wirelessly transmit recorded signals to an RZ5D real-time processor (Tucker-Davis Technologies, Alachua, FL) used for neuromodulation experiments. Synapse (Tucker-Davis Technologies, Alachua, FL), the software used to control the RZ5D, is then used to combine pulse oximetry data time-locked with neuromodulation inputs. These data are then exported and processed offline using custom MATLAB version 2019a (MathWorks, Natick, MA) script (Figure 2).

\section{Assessment of Heart Rate During Neuromodulation}

To assess whether the device would be suitable for use in neuromodulation experiments, the device had to be acceptable for the rat to wear for the duration of the stimulation. The final design was placed on the rodent's neck, and a timer was started. The session was declared over 
as soon as the device's sensor lost contact to the rodent's neck through excessive motion. The timer was then stopped.

The effects of heart rate variability were measured in a group of spontaneously hypertensive rats (SHR). These rats were receiving neuromodulation in an experiment to study the effects of VNS on development of hypertension. Under aseptic conditions, rodents were implanted with silicone-substrate cuff electrodes (Microprobes, Gaithersburg, MD) on the anterior trunk of the subdiaphragmatic vagus nerve. After recovery from surgery, animals received stimulation three times daily to study the effect of subdiaphragmatic VNS on development of hypertension. During these stimulation sessions, the off-target effects on heart rate were evaluated.

Baseline pulse oximetry data were collected before the application of vagus nerve stimulation. A cathodic leading, biphasic, current controlled stimulation was applied with an amplitude of $600 \mu \mathrm{A}$, pulse width of $500 \mu \mathrm{sec}$, at $25 \mathrm{~Hz}$. During the stimulation, heart rate was measured. These data were collected before implementing the wireless modules; however, the rest of the design remained the same. Data were analyzed offline by measuring time between peaks of the pulse oximetry waveform using MATLAB.

\section{Statistical Analysis}

To evaluate the device placement data, a one-way analysis of variance (ANOVA) using GraphPad (GraftPad Software, San Diego, CA). Post-hoc testing was completed using Tukey's HSD. Changes in instantaneous heart rate were evaluated using a two-way ANOVA using Matlab 2019a. For all tests, the level of significance was set at $\mathrm{p} \leq 0.05$. 


\section{Results}

\section{Neck Provides Best Location for Sensor Deployment}

In order to determine the best location for the device to sit on the rodent, three locations were compared for pulse oximetry placement with a chemically induced T1D rat model (Figure 3). No waveform indicative of heart rate was observed in neither the foot nor the tail. Placing the sensor on the neck resulted in a pulsatile waveform suggestive of heart rate. Analysis of the frequency domain of this waveform showed peak power at $3.56 \mathrm{~Hz}$ and $8.02 \mathrm{~Hz}$ (Figure 4). $3.56 \mathrm{~Hz}$ likely corresponds to a heart rate of $213 \mathrm{BPM}$. The peak at $8.02 \mathrm{~Hz}$ correlates to the width of the trough, lasting for approximately $125 \mathrm{~ms}$.
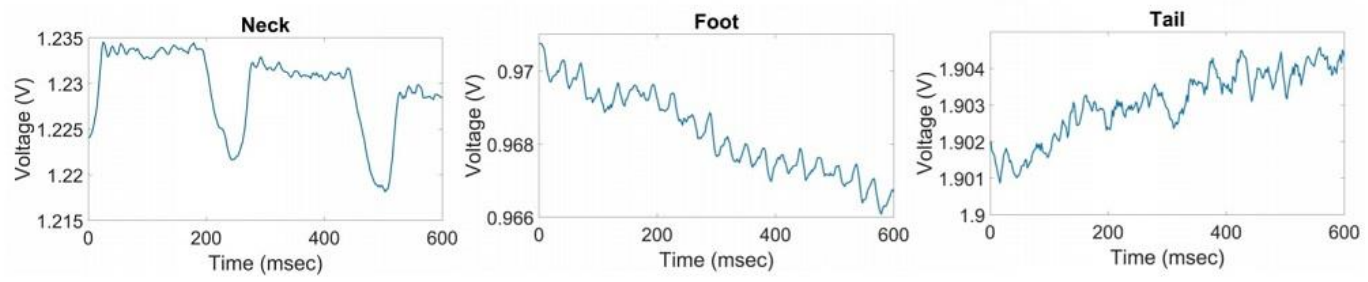

Figure 3. Recorded pulse oximetry signals from the neck (Left), foot (Middle), and tail (Right). The neck showed a pulsatile waveform with a frequency in the range of an expected heart rate while the pulsatile signals recorded from the foot and the tail waveform were absent.

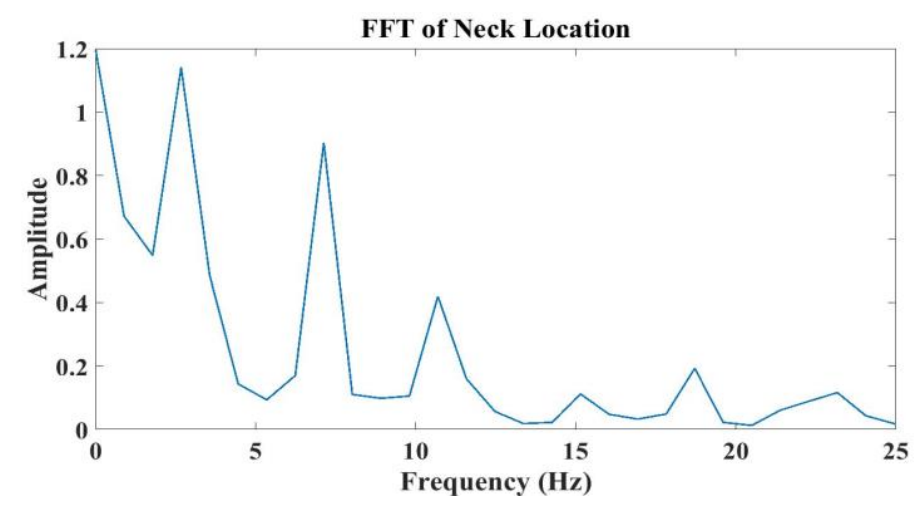

Figure 4. The neck's waveform after a FFT, showing the data in the frequency domain.

After deciding on the sensor location based on ability to record a meaningful signal, a chassis to hold the sensor was 3-D printed and the ability of the device to remain on the animal was evaluated. The average length of time for the clip to stay on the rodent had a mean of 57.2 
seconds (number of tests, $\mathrm{N}=3$ ). There was no statistical difference between the rodents tested (Figure 5).

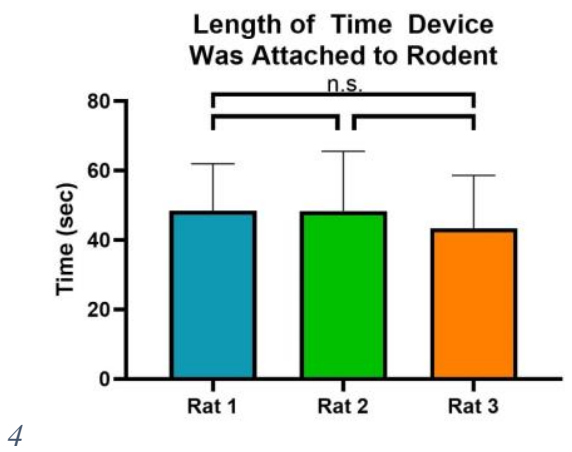

Figure 5. Mean time that the sensor was able to stay on a rodent was $57.2 \mathrm{sec}$. There were no statistical differences between rodents tested (sample size, $n=3$ ).

\section{Heart Rate Remains Constant at Stimulation Onset}

Heart rate was evaluated in a group of Spontaneously Hypertensive Rats' (SHR) before and during stimulation. Instantaneous heart rate between two consecutive beats for each pair of beats was recorded (Figure 6A). The median heart rate before stimulation was 329.28 BPM and was 326.76 BPM during stimulation. No significant difference was observed between the two

A.
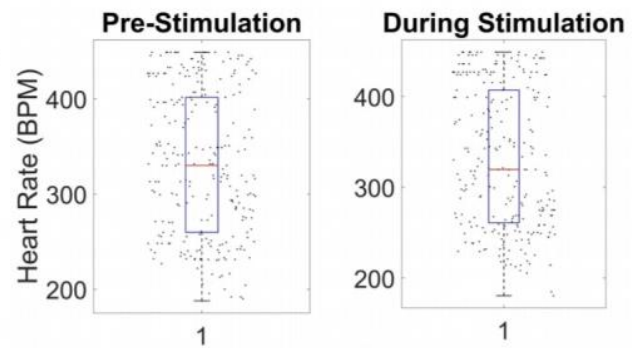

B.

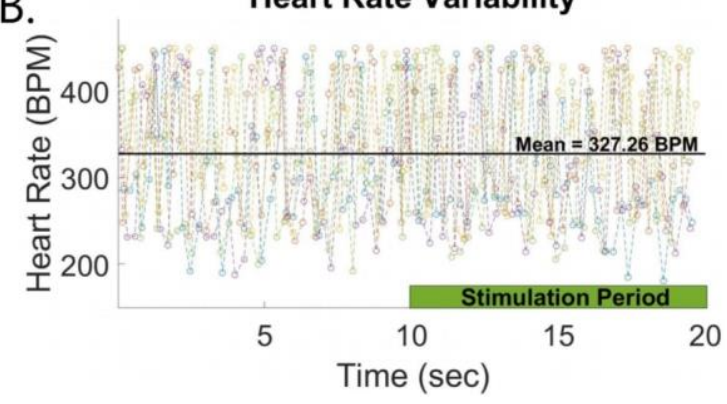

Figure 6. Heart rate variability showed similar patterns and values before and during stimulation. 
distributions. To examine if the stimulation onset had any effect on heart rate, instantaneous heart rate was plotted as a function of time (Figure 6B). The heart rate had a mean value of 327 BPM, which is normal for SHR (Lawler, 1985). There was no observed decrease in instantaneous heart rate at the onset of stimulation.

\section{Discussion}

Evaluation of this pulse oximeter was done to give labs a low-cost solution to noninvasively measure heart rate of rodents. Measuring heart rate for rodents is especially important in neuromodulation studies because it is pertinent to investigate if there are any confounding factors with the stimulus that is being applied to the nerve.

When choosing a location to implement the sensor on a small rodent, the size and weight of the device are important considerations besides just ability to detect blood flow. Since the Teensy LC and FS1000a are significant in size compared to the rats, and our device weighs $22.80 \mathrm{~g}$, the neck was the most desirable spot for this low-cost solution. It also has been observed that the rodent's neck will always provide significant blood flow for reflective pulse oximetry (Truitt, Hete, \& Starr, 2008). Our results confirmed this, showing the neck had the most consistently measurable heart rate.

A FFT of the measured pulse oximetry data showed a peak at $3.56 \mathrm{~Hz}$ corresponding to a heart rate of 214 BPM. While lower than a typically expected heart rate for rodents, this heart rate is consistent with STZ induced T1D model in rats (Howarth, 2006). Another peak was located at $8.02 \mathrm{~Hz}$, which corresponded to $125 \mathrm{~ms}$, or the amount of time between pulses.

The mean of the length of time that the clip could stay on the rodent was $57.2 \mathrm{~s}$ (Figure 6), but most neuromodulation experiments take longer than $57.2 \mathrm{~s}$. While it is not long enough to measure the whole neuromodulation study, it can be used to determine if there are any instantaneous changes to heart rate at onset of stimulation (Hotta et al., 2009). This is proven to be a long enough time for suitable heart rate measurements since the maximum change in heart rate is noted at, on average, $5 \mathrm{~s}$ after the onset of stimulation (Buschman, 2006). To capture more data, rodents can be habituated to certain objects, which can increase performance, as well as decreasing stress and anxiety (Borszcz, 1989). If the animals are habituated to the device before testing, the rodent might accept the device for longer.

During neuromodulation in the SHR hypertension model, no significant changes in heart rate variability between before and during stimulation were found. This allowed us to rule out direct 
modulation of heart rate as a potential confounding factor the study. While this experiment is designed to look at these instantaneous effects, it is possible that chronic vagus nerve stimulation may induce central plasticity and change heart rate over time. By using a subdiaphragmatic stimulation and avoiding instantaneous changes to heart rate, one can more closely examine the mechanism behind the effects of chronic VNS on prevention of the development of hypertension.

Overall, this device has been successful in recording beat to beat heart rate of conscious moving rats while being non-invasive and a low-cost solution to labs, with a cost of $11 \%$ of a used leading brand of mouse pulse oximetry device on the market. Table 1 shows the total cost of this device, $\$ 72.88$, which compared to a device found on the market similar in design, such as the MouseOx Plus (Starr LifeSciences, Oakmont, PA. A new MouseOx system has a price tag of \$5925 (Starr Life Sciences, 2020) and still is not wireless. This would save the user a significant amount of money.

Table 1. Cost of components for final design.

\begin{tabular}{c|c} 
Component & Cost \\
\hline Arduino Due & $\$ 37.40$ \\
Teensy LC & $\$ 14.38$ \\
Pulsesensor.com module & $\$ 9.95$ \\
fs1000a transceiver/ receiver & $\$ 4.95$ \\
3v cell battery (CR 2032) & $\$ 1.83$ \\
battery holder & $\$ 0.30$ \\
Perf design board & $\$ 0.92$ \\
3D printed materials $(P L A)$ & $\$ 3.15$ \\
total: & $\$ \mathbf{7 2 . 8 8}$
\end{tabular}

Future work for this device includes modifying the clip feature to have more grip to stay on the rodent longer, because most neuromodulation stimulations are done for more than the average time the clip can stay on. Another future endeavor would move the software and hardware from Arduino microcontrollers and Arduino's compiler to a smaller controller such as a PIC microcontroller. 


\section{Acknowledgements}

We thank our fellow lab members and Dr. Frank Delgado for help with preparation of the manuscript. This work was funded by the Defense Advanced Research Projects Agency (DARPA) BTO under the auspices of Dr. Douglas Weber and Dr. Eric Van Gieson through DARPA contracts Management Office (Grant No. HR0011-17-2-0019).

\section{References}

Borszcz, G. S., Cranney, J., \& Leaton, R. N. (1989). Influence of long-term sensitization on long-term habituation of the acoustic startle response in rats: Central gray lesions, preexposure, and extinction. Journal of Experimental Psychology: Animal Behavior Processes, 15(1), 54-64.

Buschman, H. P., Storm, C. J., Duncker, D. J., Verdouw, P. D., Aa, H. E. V. D., \& Kemp, P. V. D. (2006). Heart Rate Control Via Vagus Nerve Stimulation. Neuromodulation: Technology at the Neural Interface, 9(3), 214-220. doi: 10.1111/j.1525-1403.2006.00062.x

Dirr, E. W., Patel, Y. A., Lester, L., Delgado, F. \& Otto, K. J. (2019). Targeted Vagus Nerve Stimulation Does Not Disrupt Cardiac Function in the Diabetic Rat. 201941 st Annu. Int. Conf. IEEE Eng. Med. Biol. Soc. 6286-6289. doi: 10.1109/EMBC.2019.8857474

Hering, H. E. (1910). A Functional Test of Heart in Vagi in man.

Howarth, F. C., Al-Sharhan, R., Al-Hammadi, A., \& Qureshi, M. A. (2006). Effects of streptozotocininduced diabetes on action potentials in the sinoatrial node compared with other regions of the rat heart. Molecular and Cellular Biochemistry, 300(1-2), 39-46. doi: 10.1007/s11010-006-9366-5

Hotta, H., Lazar, J., Orman, R., Koizumi, K., Shiba, K., Kamran, H., \& Stewart, M. (2009). Vagus nerve stimulation-induced bradyarrhythmias in rats. Autonomic Neuroscience, 151(2), 98-105.

Lawler, J. E., Cox, R. H., Hubbard, J. W., Mitchell, V. P., Barker, G. F., Trainor, W. P., \& Sanders, B. J. (1985). Blood pressure and heart rate responses to environmental stress in the spontaneously hypertensive rat. Physiology \& Behavior, 34(6), 973-976. doi: 10.1016/0031-9384(85)90022-8

Lee, S., Shin, H., \& Hahm, C. (2016). Effective PPG sensor placement for reflected red and green light, and infrared wristband-type photoplethysmography. 2016 18th International Conference on Advanced Communication Technology (ICACT). doi: 10.1109/icact.2016.7423469

MouseOx® Plus: Clinically Validated Mouse Pulse Oximeter. (2020, April 14). Retrieved July 22, 2020, from https://www.starrlifesciences.com/product/small-animal-pulse-oximeter/

Payne, S. C., Furness, J. B., Burns, O., Sedo, A., Hyakumura, T., Shepherd, R. K., \& Fallon, J. B. (2019). Anti-inflammatory Effects of Abdominal Vagus Nerve Stimulation on Experimental Intestinal Inflammation. Frontiers in Neuroscience, 13. doi: 10.3389/fnins.2019.00418

Schachter, S. C. Vagus nerve stimulation therapy summary: Five years after FDA approval. Neurology 59, S15-S29 (2002). 
Truitt, Hete, \& Starr. (2008). Noninvasive Photoplethysmographic Sensor Platform for Mobile Animals. U.S. Patent No. 8688184B2. Washington, DC: U.S. Patent and Trademark Office.

van Ravenswaaij-Arts, C. M., Kollee, L. A., Hopman, J. C., Stoelinga, G. B., \& van Geijn, H. P. (1993). Heart rate variability. Annals of Internal Medicine, 118(6), 436-447. 